\title{
Caracterização mecânica de madeiras deterioradas em campo por meio de ultrassom e flexão estática
}

\author{
Diego Martins Stangerlin'*, Janaína De Nadai Corassa', Darci Alberto Gatto², \\ Roberto Lessa Pereira², Patrícia Aparecida Rigatto Castelo' \\ 'Universidade Federal de Mato Grosso, Campus Universitário de Sinop, Sinop, MT, Brasi \\ ${ }^{2}$ Universidade Federal de Pelotas, Pelotas, RS, Brasil \\ *Autor correspondente, e-mail: diego_stangerlin@yahoo.com.br
}

\section{Resumo}

Este estudo objetivou avaliar a resistência natural da madeira de quatro espécies florestais submetidas à deterioração em campo, por meio de ensaios não destrutivos de ultrassom e destrutivos de flexão estática. Para isso, toras de 1,2 m de comprimento de Tectona grandis (teca), Azadaractina indica (nim), Inga sp. (ingá) e Bagassa guianensis (tatajuba) foram submetidas à deterioração em ensaios de campo durante 18 meses, no município de Sinop-MT. A partir de toretes sadios, foram retirados corpos de prova para caracterização inicial dos módulos de elasticidade e de ruptura à flexão estática. Ao final do período de exposição, foram retirados corpos de prova em três posições das toras (parte enterrada, zona de afloramento e parte aérea). Os corpos de prova deteriorados foram submetidos à caracterização não destrutiva, por meio de ultrassom, em que foram determinadas as velocidades de propagação de ondas. Os corpos de prova que apresentaram as menores velocidades de propagação foram submetidos aos ensaios de flexão estática. Os resultados obtidos foram comparados àqueles dos corpos de provas sadios, de modo a caracterizar o grau de deterioração da madeira. Mediante os ensaios de ultrassom verificou-se que os corpos de prova retirados da zona de afloramento das toras apresentaram menor velocidade de propagação de ondas. Dentre as espécies estudadas, a teca e tatajuba apresentaram a maior e menor resistência natural, respectivamente.

Palavras-chave: biodeterioração, ensaio de campo, flexão estática, ultrassom

\section{Mechanical characterization of decayed wood in the field by ultrasound and static bending tests}

\begin{abstract}
This study aimed to evaluate the natural resistance of four wood species exposed to field tests using ultrasound and static bending tests. Tectona grandis (teak), Azadaractina indica (neem), Inga sp. (inga) and Bagassa guianensis (tatajuba) logs with $1.2 \mathrm{~m}$ length were subjected to field tests for 18 months, in the city of Sinop-MT. Samples were cut from healthy logs to characterize modulus of elasticity and modulus of rupture at static bending before the exposure in field test. After each period of exposure, samples were cut from the logs in three positions (in-ground contact, critical zone and above-ground). Nondestructive tests were performed using an ultrasound to determine the velocity of wave propagation in the decayed samples. Samples with lower velocities of wave propagation were subjected to the static bending tests. The results were compared with unexposed wood samples to characterize the degree of wood deterioration. Ultrasonic tests showed that samples collected from the critical zone of the logs presented lowest velocity of wave propagation. Among the species studied, teak and tatajuba wood presented the highest and the lowest natural resistance, respectively.
\end{abstract}

Keywords: biodeterioration, field test, static bending, ultrasound 


\section{Introdução}

Assim como a resistência mecânica e a estabilidade dimensional de uma madeira, as quais, comumente, são consideradas para a definição de uso de uma determinada espécie, a resistência natural à deterioração apresenta uma alta variabilidade. Essa variabilidade está relacionada intrinsecamente à madeira, em razão das diferenças na composição química (percentual de extrativos e lignina) e anatômica (porosidade) entre as espécies lenhosas, bem como ao ambiente em que a madeira será submetida (Trevisan et al., 2008; Melo et al., 2010).

DeacordocomPaesetal. (2004) eTrevisan et al. (2007), o conhecimento da resistência natural é de fundamental importância, para que seja possível recomendar o emprego de madeiras mais resistentes, de modo a minimizar possíveis custos com a substituição de peças em estágio de deterioração. Em complemento, Stangerlin et al. (2011) destacaram que os produtos à base de madeira que apresentam elevada resistência à deterioração são valorizados no mercado, ampliando o seu espectro de uso.

Para caracterização da durabilidade natural da madeira dois tipos de ensaios podem ser realizados, de laboratório ou de campo, sendo esse último mais fidedigno (Brischke \& RolfKiel, 2010), uma vez que a madeira fica sujeita à ação de organismos xilófagos (térmitas e fungos) e de fatores abióticos (temperatura e umidade do ar, precipitação pluviométrica, radiação solar, dentre outros).

De modo geral, a avaliação da durabilidade natural de uma determinada madeira pode ser realizada por meio da inspeção visual da sanidade da peça em serviço. No entanto, Trevisan et al. (2007) destacaram que a caracterização mecânica pode fornecer respostas complementares aos processos que envolvem a deterioração da madeira.

Dentre as técnicas empregadas para a caracterização mecânica da madeira destacam-se aquelas com fins não destrutivos, as quais não modificam a estrutura do material testado, facilitando o seu posterior emprego ou ainda a avaliação de madeiras em serviço. A emissão de ondas de ultrassom é uma das principais técnicas não destrutivas empregadas para a caracterização da qualidade da madeira. Dentre as suas aplicações, Gatto et al. (2012) e Weiler et al. (2013) destacaram a avaliação da integridade estrutural de peças de madeira em diferentes estágios de deterioração.

Nesse sentido, esse estudo objetivou avaliar a resistência natural da madeira de Tectona grandis (teca), Azadirachta indica (nim), Inga sp. (ingá) e Bagassa guianensis (tatajuba) submetidas a ensaios de deterioração de campo, por meio de ensaios não destrutivos de ultrassom e destrutivos de flexão estática. $\square$

\section{Material e Métodos}

Preparação das amostras

Para realização desse estudo foram utilizadas madeiras de Tectona grandis Linn. F. (teca), Azadirachta indica A. Juss. (nim), Inga sp. (ingá) e Bagassa guianensis Aub. (tatajuba), provenientes da derrubada de cinco árvores adultas, de cada espécie, com diâmetro a altura do peito de $30 \mathrm{~cm}$, aproximadamente, em povoamentos localizados no município de Sinop, região norte do estado de Mato Grosso. A madeira de teca foi proveniente, especificamente, de um plantio homogêneo de 13 anos e as demais, de um povoamento misto com diversas espécies florestais, com idade de aproximadamente 11 anos.

As árvores derrubadas foram demarcadas, adotando-se $\circ$ seguinte ordenamento: da base até 1,2 m marcou-se uma tora, e em sequência marcou-se um torete de 0,3 m (Figura 1). Procedeu-se dessa forma, até a obtenção de cinco toras e cinco toretes, de cada espécie florestal, para instalação dos ensaios de deterioração em campo (utilizando as toras) e confecção de corpos de prova sadios (utilizando os toretes).

A partir dos toretes, de cada espécie, foram confeccionados cinco corpos de prova de $2 \times 2 \times 20 \mathrm{~cm}$ (maior dimensão no sentido axial), os quais foram colocados em câmara climática, com temperatura de $20^{\circ} \mathrm{C}$ e umidade relativa de $65 \%$, até atingirem um teor de umidade de equilíbrio de $12 \%$. Após a climatização da madeira, determinou-se a massa específica aparente dos corpos de prova, por meio da relação entre massa e volume. 


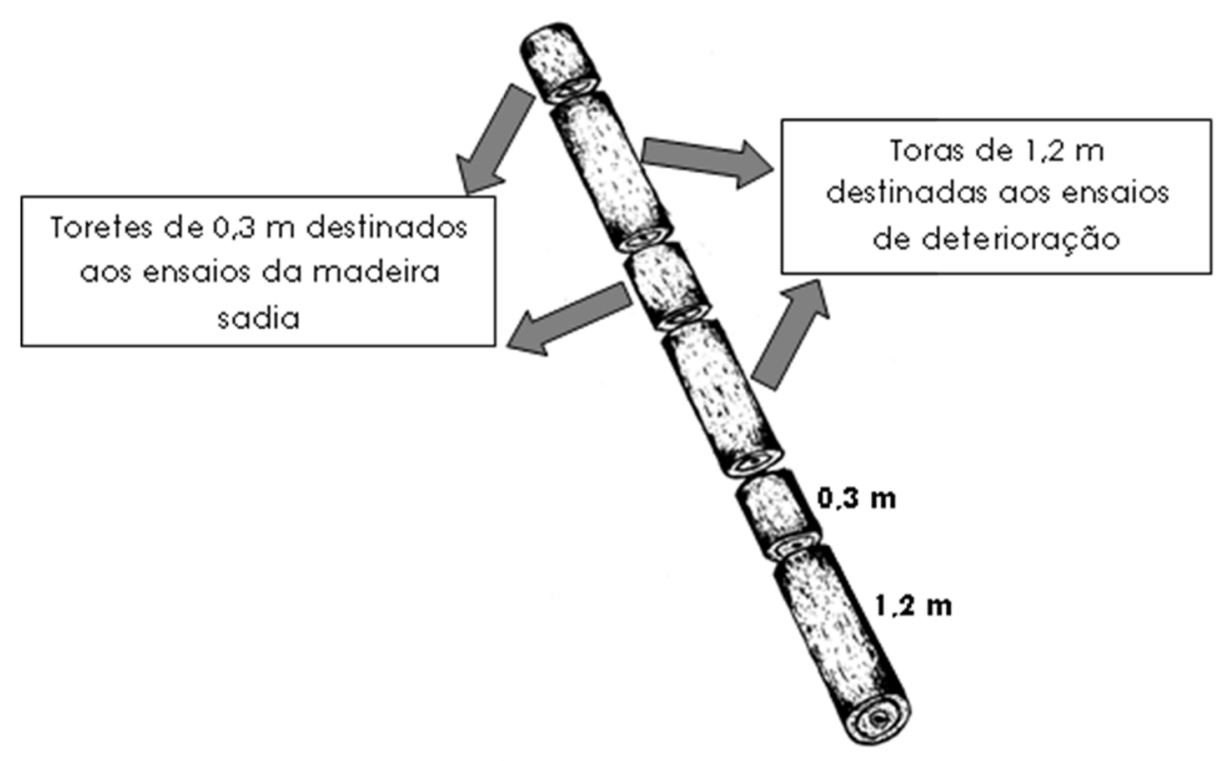

Figura 1. Preparação das amostras a partir das árvores derrubadas.

Instalação dos ensaios de deterioração de campo

Os ensaios de deterioração foram instalados em uma área experimental situada a 11050'53" de latitude sul e 55³8'57" de longitude Oeste, com altitude média de 380 m, no município de Sinop, Mato Grosso. O local de instalação encontra-se em uma Área de Preservação Permanente com vegetação florestal em estágio secundário de regeneração. O clima da região, segundo a classificação de Köppen, é do tipo "Aw" tropical, quente e úmido, caracterizado pela presença de duas estações bem definidas, uma chuvosa (entre os meses de outubro a abril) e outra de seca (entre os meses de maio a setembro).

As toras, sem serem descascadas, foram enterradas verticalmente no solo a uma profundidade de $30 \mathrm{~cm}$, ficando $90 \mathrm{~cm}$ acima da linha de afloramento, de modo a proporcionar a exposição tanto dos agentes bióticos quanto abióticos do local (Figura 2). A disposição das toras foi definida pela divisão em quatro blocos casualizados, contendo cinco toras em cada, sendo o espaçamento de $1 \mathrm{~m}$ entre as toras e de 1,5 m entre os blocos.

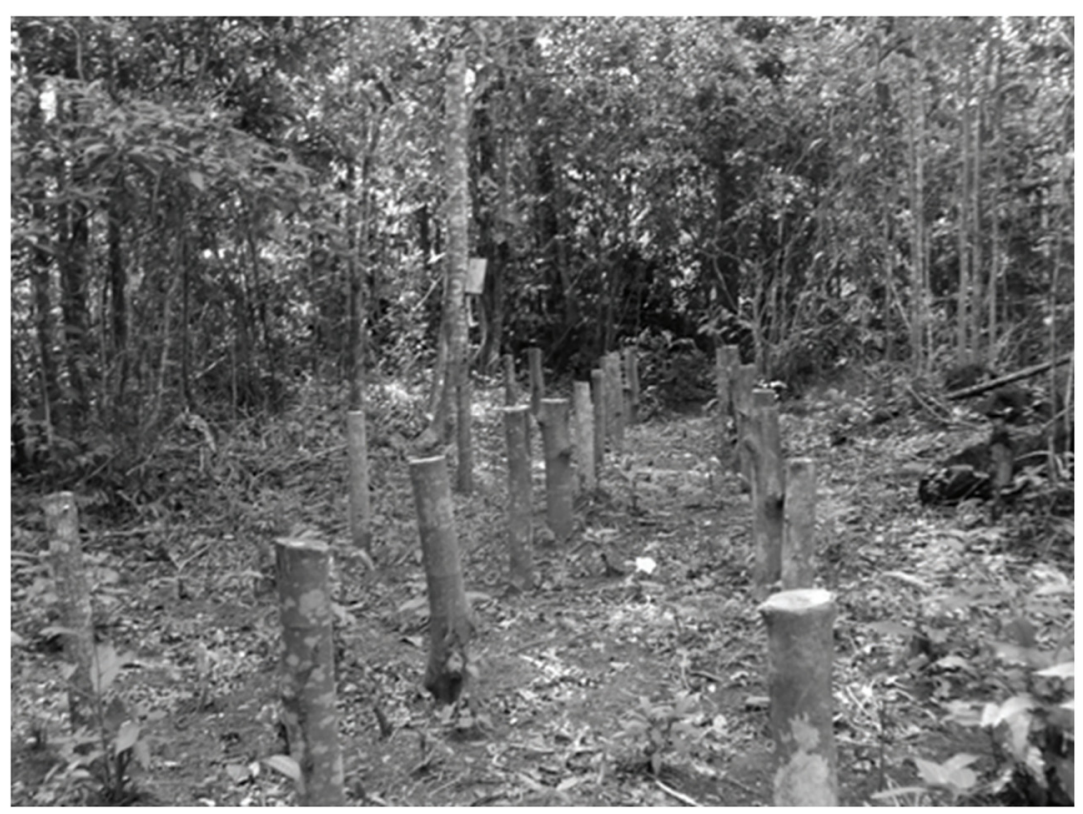

Figura 2. Preparação das amostras a partir das árvores derrubadas. 
Após 18 meses de instalação dos ensaios de deterioração, as toras foram retiradas do ambiente e submetidas ao desdobro para obtenção, quando possível, de três corpos de prova, em três posições distintas (sentido axial), de cada tora, com dimensões de $2 \times 2$ $x 20 \mathrm{~cm}$ (maior dimensão no sentido axial). A sistematização de retirada dos corpos de prova teve como premissa uma amostragem que representasse toda uma possível variação de ataque de organismos xilófagos. Nesse sentido foram retirados corpos de prova na base (parte totalmente enterrada), na região de afloramento (transição da parte enterrada com a parte sem contato com o solo) e na parte fora do contato com o solo. Em seguida, os corpos de prova foram submetidos à climatização até atingirem um teor de umidade de equilíbrio de $12 \%$.

Caracterização não destrutiva e destrutiva das madeiras sadias e deterioradas

Os corpos de prova deteriorados foram submetidos aos ensaios não destrutivos, para análise da integridade estrutural das diferentes posições das toras, com auxílio de um equipamento emissor de ondas ultrassônicas. Esse equipamento era dotado de transdutores de pontos secos com frequência de aproximadamente $50 \mathrm{kHz}$, sendo obtido diretamente o tempo de propagação das ondas, em microssegundos (? s).

Os transdutores foram posicionados nas extremidades dos corpos de prova, considerando o sentido axial (método direto), sendo realizadas duas leituras do tempo de propagação das ondas. A partir da razão entre a média do tempo de propagação das ondas e a distância percorrida (comprimento de cada corpo de prova), calculou-se a velocidade de propagação das ondas (m/s).

Os corpos de prova deteriorados que apresentaram as menores velocidades de propagação das ondas de ultrassom foram ensaiados destrutivamente à flexão estática. Essa seleção teve como intuito caracterizar a zona axial da tora que apresentasse a máxima deterioração, tanto pelos agentes bióticos quanto abióticos. Os ensaios de flexão estática foram realizados com auxílio de uma máquina universal de ensaios, sendo obtidos os módulos de elasticidade e de ruptura, seguindo padronizações estabelecidas na norma D143 (ASTM, 1994). Os resultados desses testes foram comparados àqueles obtidos para os corpos de prova sadios, provenientes dos toretes.

Os resultados de velocidade de propagação de ondas de ultrassom foram submetidos à análise de variância considerando os fatores espécie florestal (em quatro níveis) e posição axial (em três níveis). Por sua vez, para a análise de variância dos resultados de resistência mecânica à flexão estática (módulos de elasticidade e de ruptura) consideraram-se os fatores espécie florestal (em quatro níveis) e sanidade (em dois níveis). Os fatores tidos como significativos pelo teste $F(p \leq 0,05)$ foram desdobrados e analisados, separadamente, pelo teste de comparação de médias DMS (diferença mínima significativa) de Fischer ( $p \leq$ $0,05)$.

\section{Resultados e Discussão}

Na Tabela 1 pode-se observar que, independente da posição axial (posição de retirada dos corpos de prova nas toras após os ensaios de deterioração), a velocidade de propagação das ondas de ultrassom na madeira de teca foi maior em relação às demais espécies expostas à deterioração. Conforme descrito por Carrasco \& Azevedo Júnior (2003), Calegari et al. (2007), Calegari et al. (2008) e Gatto et al. (2012), fatores intrínsecos da madeira como as propriedades anatômicas (dimensões de fibras e frequência de raios), físicas (massa específica e teor de umidade) e morfológicas (tipos de lenho e ângulo da grã), bem como o grau de deterioração, influenciam diretamente na propagação das ondas de ultrassom.

Em relação à posição axial, apesar das menores médias de propagação de ondas de ultrassom serem observadas nos corpos de prova extraídos da zona de afloramento das toras, não foi verificada diferença estatística entre as diferentes posições. Brischke \& Rolf-Kiel (2010) e Schneid et al. (2011) descreveram que numa peça roliça de madeira em serviço a zona de afloramento apresenta o maior grau de deterioração. 
Tabela 1. Valores médios da velocidade de propagação das ondas de ultrassom nas quatro madeiras expostas à deterioração em campo.

\begin{tabular}{cccc}
\hline \multirow{2}{*}{ Espécies } & \multicolumn{3}{c}{ Posição Axial } \\
\cline { 2 - 4 } & Parte Enterrada & Zona de Afloramento & Parte Aérea \\
\hline \multirow{2}{*}{ Teca } & $2804,68 \mathrm{~b} \mathrm{~A}^{1}$ & $2710,78 \mathrm{~b} \mathrm{~A}$ & $2826,86 \mathrm{~b} \mathrm{~A}$ \\
& $( \pm 47,34)^{2}$ & $( \pm 106,64)$ & $( \pm 59,99)$ \\
Ingá & $2520,91 \mathrm{a} \mathrm{A}$ & $2486,88 \mathrm{aA}$ & $2653,36 \mathrm{ab} \mathrm{A}$ \\
& $( \pm 165,02)$ & $( \pm 279,37)$ & $( \pm 135,58)$ \\
Tatajuba & $2531,84 \mathrm{a} \mathrm{A}$ & $2525,26 \mathrm{ab} \mathrm{A}$ & $2623,31 \mathrm{ab} \mathrm{A}$ \\
& $( \pm 22,43)$ & $( \pm 31,90)$ & $( \pm 74,58)$ \\
Nim & $2457,18 \mathrm{a} \mathrm{A}$ & $2424,80 \mathrm{a} \mathrm{A}$ & $2494,96 \mathrm{a} \mathrm{A}$ \\
& $( \pm 17,25)$ & $( \pm 113,06)$ & $( \pm 19,82)$
\end{tabular}

de erro. ${ }^{2}$ Valores entre parênteses são relativos ao desvio padrão.

De acordo com Oliveira et al. (1986), madeiras em serviço, como no caso de toras utilizadas como moirões, apresentam diferentes graus de deterioração em função da posição axial: na parte superior, sem contato com o solo, a deterioração está relacionada em grande parte aos fatores abióticos, apesar de haver também ataque de organismos xilófagos; a zona de afloramento é a mais crítica, visto que a mesma apresenta elevada umidade e teor de oxigenação, de modo a favorecer o ataque de diferentes xilófagos, em especial por fungos apodrecedores; na base, parte totalmente enterrada, também se verifica uma elevada umidade, entretanto o teor de oxigenação é reduzido, de modo que a deterioração fica restrita a ação de bactérias anaeróbicas.

Nesse sentido, a menor velocidade de propagação das ondas de ultrassom nos corpos de prova retirados da zona de afloramento das toras é justificada em razão da sua maior suscetibilidade à deterioração, quando em contato com o solo. Gatto et al. (2012) e Weiler et al. (2013) afirmaram que a deterioração biológica afeta a estrutura e ultra-estrutura da madeira, proporcionando a descontinuidade do meio para a propagação das ondas de ultrassom. Essa descontinuidade está relacionada à presença de zonas de apodrecimento intenso ou de cavidades formadas por insetos. Em complemento, Mattos et al. (2013) enfatizaram que a perda de compactação do material celulósico é refletida na redução da velocidade de propagação das ondas de ultrassom.

Ao analisar a média geral da velocidade de propagação das ondas de ultrassom, independente da espécie, verificou-se um valor de aproximadamente $2600 \mathrm{~m} / \mathrm{s}$. Considerando um valor médio da velocidade das ondas de ultrassom de $5000 \mathrm{~m} / \mathrm{s}$, pelo método direto, conforme relatado por Stangerlin et al. (2010), pode-se estimar que houve uma atenuação da propagação de cerca de 52\%. Shaji et al. (2000), ao compararem as velocidades de propagação das ondas de ultrassom obtidas em peças sadias e deterioradas, atribuíram que uma atenuação acima de $30 \%$ indica que uma peça de madeira necessita de alguma intervenção, como por exemplo, tratamento curativo ou substituição por outra peça sadia. Sendo assim, destaca-se que as espécies avaliadas no presente estudo, caso estivessem em uso estrutural, necessitariam de substituição devido à intensa deterioração biológica.

Na Tabela 2 pode-se observar que os módulos de elasticidade e de ruptura à flexão estática dos corpos de prova retirados da zona de afloramento das toras foram significativamente influenciados em razão da exposição aos ensaios de deterioração em campo. De acordo com Wilcox (1978), os ensaios de flexão estática, bem como os de flexão dinâmica e dureza, são os mais indicados na caracterização da deterioração da madeira, nesse sentido os resultados encontrados corroboram com a literatura em virtude da sensibilidade desses ensaios.

Com relação à flexão estática, Trevisan et al. (2007) e Weiler et al. (2013) verificaram que $\circ$ módulo de ruptura apresenta uma maior redução em comparação ao módulo de elasticidade, tornando-o assim mais eficaz na caracterização da deterioração biológica. Entretanto, no presente estudo não se verificou 
esse comportamento em todas as espécies, sendo esse constatado apenas para as madeiras de tatajuba e teca.

Ao analisar o comportamento das espécies lenhosas, pode-se constatar que a maior massa específica da madeira de tatajuba não contribuiu para minimizar a deterioração da madeira, uma vez que a mesma apresentou a maior perda de resistência mecânica, cerca de $81,69 \%$ e $58,93 \%$ para os módulos de ruptura e elasticidade, respectivamente. Entretanto,
Oliveira et al. (2005), Paes et al. (2007) e Stangerlin et al. (2013) destacaram que a resistência natural de uma determinada madeira não deve ser associada apenas aos parâmetros quantitativos como a massa específica e o teor de extrativos, mas principalmente com os parâmetros qualitativos, em que se destacam o grau de toxidez de determinados extrativos e a forma de distribuição dos constituintes de alto e baixo peso molecular.

Tabela 2. Valores médios de massa específica aparente a 12\% de umidade e os módulos de elasticidade e de ruptura à flexão estática das madeiras sadias e deterioradas

\begin{tabular}{cccccc}
\hline Espécies & MEap $\left(\mathrm{g} / \mathrm{cm}^{3}\right)$ & $\mathrm{fM} \mathrm{sd}(\mathrm{MPa})$ & $\mathrm{fM} \mathrm{d}(\mathrm{MPa})$ & $\mathrm{EMO} \mathrm{sd}(\mathrm{MPa})$ & $\mathrm{EM0} \mathrm{d}(\mathrm{MPa})$ \\
\hline \multirow{2}{*}{ Teca } & $0,64 \mathrm{~b}^{1}$ & $140,80 \mathrm{~b} \mathrm{~A}$ & $69,43 \mathrm{a} \mathrm{B}$ & $10630 \mathrm{c} \mathrm{A}$ & $5426 \mathrm{a} \mathrm{B}$ \\
& $( \pm 0,011)^{2}$ & $( \pm 0,48)$ & $( \pm 22,88)$ & $( \pm 57,19)$ & $( \pm 1807)$ \\
Ingá & $0,62 \mathrm{c}$ & $136,33 \mathrm{~b} \mathrm{~A}$ & $44,97 \mathrm{c} \mathrm{B}$ & $13260 \mathrm{a} \mathrm{A}$ & $3787 \mathrm{c} \mathrm{B}$ \\
& $( \pm 0,001)$ & $( \pm 0,35)$ & $( \pm 23,79)$ & $( \pm 96,53)$ & $( \pm 1735)$ \\
Tatajuba & $0,70 \mathrm{a}$ & $221,39 \mathrm{a} \mathrm{A}$ & $40,53 \mathrm{c} \mathrm{B}$ & $11440 \mathrm{~b} \mathrm{~A}$ & $4698 \mathrm{~b} \mathrm{~B}$ \\
& $( \pm 0,0068)$ & $( \pm 0,86)$ & $( \pm 26,83)$ & $( \pm 91,67)$ & $( \pm 1923)$ \\
Nim & $0,56 \mathrm{~d}$ & $106,79 \mathrm{c} \mathrm{A}$ & $51,86 \mathrm{~b} \mathrm{~B}$ & $11510 \mathrm{~b} \mathrm{~A}$ & $4602 \mathrm{~b} \mathrm{~B}$ \\
& $( \pm 0,0091)$ & $( \pm 0,46)$ & $( \pm 23,46)$ & $( \pm 205,14)$ & $( \pm 1692)$ \\
\hline
\end{tabular}

em que: MEap = Massa específica aparente a 12\% de umidade; fM sd = Módulo de ruptura à flexão estática da madeira sadia; fM d = Módulo de ruptura à flexão estática da madeira deteriorada; $E M O$ sd = Módulo de elasticidade à flexão estática da madeira sadia; EMO d = Módulo de elasticidade à flexão estática da madeira deteriorada. 'Médias seguidas por uma mesma letra maiúscula na horizontal ou por uma mesma letra minúscula na vertical, não diferem estatisticamente entre si a $5 \%$ de probabilidade de erro. ${ }^{2}$ Valores entre parênteses são relativos ao desvio padrão.

Assim como a tatajuba, a madeira de ingá também apresentou considerável perda de resistência mecânica, aproximadamente $67,01 \%$ e $71,44 \%$ para os módulos de ruptura e elasticidade, respectivamente. Segundo Corassa et al. (2013 e 2014) a madeira de ingá apresenta compostos voláteis que atraem os agentes xilófagos, em destaque para os térmitas. Trevisan et al. (2008) verificaram para madeira do mesmo gênero quando submetida à deterioração em campo, uma alta suscetibilidade ao ataque de coleópteros, com destaque para Euplatypus parallelus e Teloplatypus ratzeburgi.

Por sua vez, as madeiras de teca e nim foram as que apresentaram as menores perdas de resistência mecânica, sendo os decréscimos nos módulos de ruptura e de elasticidade, de cerca de $50,68 \%$ e $48,95 \%$ para a primeira e de $51,44 \%$ e $60,01 \%$ para a segunda. Para a madeira de nim, tal resultado pode ser justificável em razão do seu cerne ser rico em taninos (composto fenólico) e sais inorgânicos de cálcio, potássio e ferro (Araújo et al., 2000). Além disso, destacase a presença na casca do nim, em pequena escala, da azadiractina, a qual se caracteriza por ser um repelente a ação de insetos (Corassa et al., 2014). De acordo com Motta et al. (2014), a resistência natural da madeira de teca está relacionada à presença de flavonóides, em especial a tectoquinona. Corassa et al. (2013) também mencionaram a presença de uma substância denominada caucho, que reduz a absorção de água e, consequentemente o desenvolvimento de fungos xilófagos.

\section{Conclusões}

Os ensaios não destrutivos de ultrassom demonstraram que os corpos de prova retirados da zona de afloramento das toras apresentaram menor velocidade de propagação de ondas.

Dentre as espécies estudadas, a madeira de teca foi a que apresentou a maior resistência natural, em razão da menor alteração nos módulos de elasticidade e de ruptura, por outro lado a madeira de tatajuba foi a mais suscetível à deterioração.

\section{Referências}

Araújo, L.V.C., Rodriguez, L.C.E., Paes, J.B. 2000. Características físico-químicas e energéticas da madeira de nim indiano. Scientia Florestalis 57: 
153-159.

ASTM. American Society for Testing and Materials. 1994. D143: Standard methods of testing small clear specimens of timber. ASTM Standards, Philadelphia. p.24-65.

Brischke, C., Rolf-Kiel, H. 2010. Durability of European oak (Quercus spp.) in ground contact - A case study on fence posts in service. Holz als Roh- und Werkstoff 68: 129-137.

Calegari, L., Stangerlin, D.M., Santini, E.J., Haselein, C.R., Longhi, S.J., Carmo, P.I.O., Silva Filho, L.C.P., Gatto, D.A. 2007. Monitoramento do teor de umidade de madeiras de Pinus elliottii Engelm. e Eucalyptus grandis W. Hill ex Maiden, sob diferentes temperaturas de secagem, através do ultra-som. Ciência Florestal 17: 399-408.

Calegari, L., Stangerlin, D.M., Santini, E.J., Haseilen, C.R., Gatto, D.A., Carmo, P.I.O., Silva Filho, L.C.P. 2008. Avaliação de alguns fatores influentes na velocidade ultra-sônica na madeira. Floresta 38: 607-615

Carrasco, E.V.M., Azevedo Júnior, A.P. 2003. Avaliação não destrutiva de propriedades mecânicas de madeiras através de ultra-som fundamentos físicos e resultados experimentais. Cerne 9: 178-191.

Corassa, J.N., Castelo, P.A.R., Stangerlin, D.M., Magistrali, I.C. 2013. Durabilidade natural da madeira de quatro espécies florestais em ensaios de deterioração em campo. Ciência da Madeira 4: 108-117.

Corassa, J.N., Pires, E.M., Andrade Neto, V.R., Tariga, T.C. 2014. Térmitas associados à degradação de cinco espécies florestais em campo de apodrecimento. Floresta e Ambiente 21: 78-84.

Gatto, D.A., Gonçalvez, M.R.F., Mattos, B.D., Calegari, L., Stangerlin, D.M. 2012. Estimativa da deterioração da madeira de assoalho de prédio histórico por meio de ondas ultrassônicas. Cerne 18: 651-656.

Mattos, B.D., Gatto, D.A, Cademartori, P.H.G, Stangerlin, D.M., Beltrame, R. 2013. Durabilidade a campo da madeira de três espécies de Eucalyptus tratadas por imersão simples. Agrária 8: 648-655.

Melo, R.R.; Stangerlin, D.M., Santini, E.J., Haselein, C.R., Gatto, D.A., Susin. F. 2010. Durabilidade natural da madeira de três espécies florestais em ensaios de campo. Ciência Florestal 20: 357-365.

Motta, J.P., Oliveira, J.T.S., Paes, J.B., Alves, R.C. Dambroz, G.B.V. 2014. Resistência natural da madeira de Tectona grandis em ensaio de laboratório. Ciência Rural 43: 1393-1398.
Oliveira, A.M.F., Lelis, A.T., Lepage, E.S., Carballera Lopez, G.A., Oliveira, L.C.S., Canedo, M.D., Milano, S. 1986. Agentes destruidores da madeira. In: Lepage, E.S. (ed.) Manual de preservação de madeiras. IPT, São Paulo. p.99-278.

Oliveira, J.T.S., Souza, L.C., Della Lucia, R.M., Souza Júnior, W.P. 2005. Influência dos extrativos na resistência ao apodrecimento de seis espécies de madeira. Árvore 29: 819-826.

Paes, J.B., Melo, R.R., Lima, C.R. 2007. Resistência natural de sete madeiras a fungos e cupins xilófagos em condições de laboratório. Cerne 13: 160-169.

Paes, J.B., Morais, V.M., Lima, C.R. 2004. Resistência natural de nove madeiras do semiárido brasileiro a fungos xilófagos em condições de laboratório. Árvore 28: 275-282.

Schneid, E., Gatto, D.A., Cademartori, P.H.G., Hamm, L.G., Stangerlin, D.M. 2011. Avaliação não destrutiva de postes de madeira utilizados na rede elétrica da região metropolitana de Porto Alegre. Ciência da Madeira 2: 43-52.

Shaji, T., Somayaji, S., Mathews, M.S. 2000. Ultrasonic pulse velocity technique for inspection and evaluation of timber. Journal of Materials in Civil Engineering 12: 180-185.

Stangerlin, D.M., Gonçalez, J.C., Gonçalves, R., Santini, E.J., Calegari, L., Gatto, D.A., Melo, R.R. 2010. Avaliação de tipos de ondas geradas por dois modelos de transdutores para determinação do módulo de elasticidade dinâmico. Floresta 40: 691-700. 
Stangerlin, D.M., Melo, R.R., Garlet, A., Gatto, D.A. 2011. Durabilidade natural de painéis aglomerados confeccionados com Eucalyptus grandis e Bambusa vulgaris em ensaio de apodrecimento acelerado. Ciência Rural 41: 1369-1374.

Stangerlin, D.M., Costa, A.F., Garlet, A., Pastore, T.C.M. 2013. Resistência natural da madeira de três espécies amazônicas submetidas ao ataque de fungos apodrecedores. Ciência da Madeira 4: 15-32.

Trevisan, H., Tieppo, F.M.M., Carvalho, A.G. 2008. Degradação natural de toras de cinco espécies florestais em dois ambientes. Floresta 38: 33-40.

Trevisan H., Tieppo F.M.M., Carvalho A.G., Lelis, R.C.C. 2007. Avaliação de propriedades físicas e mecânicas da madeira de cinco espécies florestais em função da deterioração em dois ambientes. Árvore 31: 93-101.

Weiler, M., Missio, A.L., Gatto, D.A., Güths, W.G. 2013. Nondestructive evaluation of wood decayed by xylophagous organisms. Materials Research 16: 1203-1213.

Wilcox, W.W. 1978. Review of literature on the effects of early stages of decay on wood strength. Wood and Fiber Science 9: 252-257. 